\title{
Diacronie
}

Studi di Storia Contemporanea

$N^{\circ} 33,1 \mid 2018$

Guerra e pace

\section{Arturo Marzano, Storia dei sionismi. Lo Stato degli Ebrei da Herzl a oggi}

\section{Elisa Tizzoni}

\section{Q OpenEdition}

Journals

Edizione digitale

URL: http://journals.openedition.org/diacronie/7441

DOI: $10.4000 /$ diacronie.7441

ISSN: 2038-0925

Editore

Association culturelle Diacronie

Notizia bibliografica digitale

Elisa Tizzoni, « Arturo Marzano, Storia dei sionismi. Lo Stato degli Ebrei da Herzl a oggi », Diacronie

[Online], $N^{\circ}$ 33, 1 | 2018, documento 7, Messo online il 29 mars 2018, consultato il 24 septembre 2020.

URL : http://journals.openedition.org/diacronie/7441 ; DOI : https://doi.org/10.4000/diacronie.7441 


\section{Diacronie}

Studi di Storia Contemporanea

\section{$33,1 / 2018$}

Guerra e pace: declinazioni politiche, sociali e culturali del conflitto in età contemporanea

\section{RECENSIONE: Arturo MARZANO, Storia dei sionismi. Lo Stato degli Ebrei da Herzl a oggi, Roma, Carocci, 2017, 256 pp.}

\section{A cura di Elisa TIZZONI}

Per citare questo articolo:

TIZZONI, Elisa, «RECENSIONE: Arturo MARZANO, Storia dei sionismi. Lo Stato degli Ebrei da Herzl a oggi, Roma, Carocci, 2017, 256 pp.», Diacronie. Studi di Storia Contemporanea : Guerra e pace: declinazioni politiche, sociali e culturali del conflitto in età contemporanea, 33, 1/2018, 29/03/2018,

URL: <http://www.studistorici.com/2018/03/29/tizzoni_numero_33/ >

Diacronie Studi di Storia Contemporanea $\rightarrow$ http://www.diacronie.it Rivista storica online. Uscita trimestrale.

redazione.diacronie@hotmail.it

Comitato di direzione: Naor Ben-Yehoyada - João Fábio Bertonha - Christopher Denis-Delacour - Maximiliano Fuentes Codera Anders Granås Kjøstvedt - John Paul Newman - Deborah Paci - Niccolò Pianciola - Spyridon Ploumidis - Wilko Graf Von Hardenberg

Comitato di redazione: Jacopo Bassi - Luca Bufarale - Gianluca Canè - Luca G. Manenti - Fausto Pietrancosta - Alessandro Salvador - Matteo Tomasoni - Luca Zuccolo 


\title{
7/ RECENSIONE: Arturo MARZANO, Storia dei sionismi. Lo Stato degli Ebrei da Herzl a oggi, Roma, Carocci, 2017, 256 pp.
}

\author{
A cura di Elisa TIZZONI
}

In un momento storico di forte instabilità per lo Stato di Israele, in parte conseguenza di scelte controverse operate dalle sue classi dirigenti, la Storia dei sionismi di Arturo Marzano ${ }^{1}$ offre un contributo di grande valore alla comprensione delle fratture che ancora oggi impediscono la convivenza pacifica tra popolazione ebraica ed araba, innescando una serie di reazioni a catena che si estendono ben al di là dello scacchiere mediorientale.

La pubblicazione di quest'opera, come sottolineato dallo stesso autore, avviene in un anno, il 2017, nel quale ricorrono gli anniversari di alcuni degli eventi più significativi per la storia del sionismo (tra i quali il centenario della dichiarazione Balfour ${ }^{2}$ ), che hanno suscitato interesse per la storia di questo movimento ma anche forti polemiche contro alcune delle iniziative assunte per commemorare queste ricorrenze ${ }^{3}$.

Il testo, che raccoglie i risultati di oltre vent'anni di ricerche da parte dell'autore, affronta l'evoluzione del sionismo padroneggiando concetti elaborati dalla scienza politica, dalla sociologia, dalla filosofia ${ }^{4}$, mantenendo una costante attenzione alla concretezza delle vicende storiche che, probabilmente, deriva dell'esperienza professionale ed umana maturata da Marzano in Medio Oriente come inviato di una nota associazione internazionale impegnata nella cooperazione internazionale.

\footnotetext{
${ }^{1}$ MARZANO, Arturo, Storia dei sionismi. Lo Stato degli ebrei da Herzl a oggi, Roma, Carocci, 2017. Arturo Marzano è attualmente professore associato nel settore disciplinare Storia e Istituzioni dell'Asia presso il Dipartimento di Civiltà e forme del sapere dell'Università di Pisa.

${ }^{2}$ Nel 2017 ricorrono i 120 anni dal primo congresso sionista di Basilea e i 70 anni dalla Risoluzione n. 181 delle Nazioni Unite con la quale si adottava un piano per la nascita di uno stato ebraico in Palestina, oltre ad altri anniversari citati puntualmente nel volume.

${ }^{3}$ Sulle polemiche associate alla commemorazione del Centenario della Dichiarazione Balfour si veda BLACK, Ian, «The Contested Centenary of Britain's 'Calamitous Promise'», in The Guardian, 17 ottobre 2017, URL: < http://www.theguardian.com > [consultato il 7 dicembre 2017].

${ }^{4}$ BIDUSSA, David, Il sionismo politico, Milano, Unicopli, 1993; BENSOUSSAN, Georges, Il sionismo. Una storia politica e intellettuale 1860-1940, Torino, Einaudi, 2007.
} 
L'opera, dunque, propone una rilettura complessiva e plurale del movimento che ha condotto alla nascita dello Stato di Israele e ne ha condizionato successivamente le vicende, individuando contenuti ideologici, strumenti di comunicazione e lotta politica, gruppi sociali e aree territoriali di riferimento, personalità di spicco dei diversi sionismi (la scelta della forma plurale nel titolo non è casuale). La scelta di adottare un orizzonte temporale che giunge sino ai giorni nostri definisce ulteriormente l'originalità del volume nei confronti della gran parte delle storie del sionismo pubblicate negli scorsi decenni, che concludono la trattazione con la nascita dello stato di Israele ${ }^{5}$, ignorando il fatto che «il sionismo [...] non solo ha continuato ad esistere dopo di allora, ma è anche profondamente mutato nei decenni successivi» ${ }^{6}$.

I primi capitoli prendono in esame i decenni centrali del XIX secolo, quando si sviluppò un vivace dibattito sul futuro della popolazione ebraica, che contrapponeva "assimilazione" e "nazionalismo ebraico" e, all'interno di questa seconda opzione, si interrogava su un possibile ritorno ad Eretz Israel (la "Terra di Israele", definizione carica di complessi significati culturali e

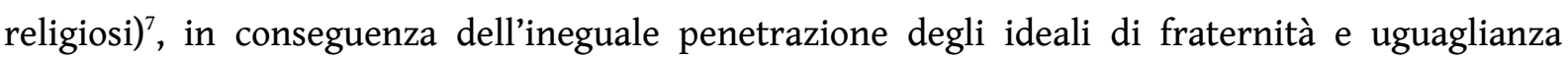
scaturiti dalla Rivoluzione francese nei diversi paesi di residenza delle comunità ebraiche.

Come ci aspetteremmo, l'autore dedica particolare attenzione alla figura di Theodor Herzl e al suo operato e ricostruisce con chiarezza le intricate vicende che precedettero il Congresso di Basilea (1897) e, con esso, l'affermazione del "sionismo politico", secondo il quale «il consenso dei governi era prioritario»" ${ }^{8}$, nei confronti di altri movimenti (il più rilevante dei quali fu il Bund ${ }^{9}$ ) e correnti interne (in particolare il cosiddetto "sionismo pratico", a favore di un'immediata ed effettiva occupazione dei territori di Eretz Israel).

La trattazione prosegue occupandosi delle conseguenze della seconda aliyah ${ }^{10}$ (1904-1914), esaminando in che modo il sionismo fu influenzato dal pensiero socialista russo, data la pregressa militanza di numerosi aderenti nelle fila di movimenti operai dell'est europeo, e ricapitolando i

\footnotetext{
${ }^{5}$ É il caso di opere note ed ampiamente utilizzate a scopo didattico oltre che di ricerca, tra le quali HERTZBERG, Arthur (edited by), The Zionist Idea: A Historical Analysis and Reader, Philadelphia, The Jewish Publication Society, 1959; LAQUEUR, Walter, A History of Zionism, New York, Schocken Books, 1972; AVINERI, Shlomo, The Making of Modern Zionism: Intellectual Origins of the Jewish State, New York, Basic Books, 1981; DIECKHOFF, Alain, L'invention d'une nation: Israël et la modernité politique, Parigi, Gallimard, 1993; GOLDBERG, David J., Verso la terra promessa. Storia del pensiero sionista, Bologna, il Mulino, 1999 [Ed. originale: Towards the Promised Land. A History of Zionist Thought from its origins to the modern state of Israel, London, Penguin, 1996]; BRENNER, Michael, Breve storia del sionismo, Roma-Bari, Laterza, 2003 [Ed. originale: Geschichte des Zionismus, München, Beck TB, 2002].

${ }^{6}$ MARZANO, Arturo, Storia dei sionismi, cit., p. 11.

${ }^{7}$ BENSOUSSAN, Georges, op. cit.

${ }^{8}$ MARZANO, Arturo, Storia dei sionismi, cit., p. 4.

${ }^{9}$ Con il termine Bund si indica comunemente la Lega generale dei lavoratori ebrei di Lituania, Russia e Polonia, filiazione del movimento operaio russo di orientamento anti-sionista (per un quadro generale della storiografia in materia si veda WOLFF, Frank, «Historiography on the General Jewish Labor Bund: Traditions, Tendencies and Expectations», in Medaon, 4/2009, URL: < http://www.medaon.de/pdf/M_Wolff4-2009.pdf > [consultato il 7 dicembre 2017].

${ }^{10}$ Trascrizione del termine ebraico con il quale si indica l'immigrazione in Palestina.
} 
termini principali del dibattito storiografico sull'atteggiamento "coloniale" che la comunità ebraica immigrata in Palestina avrebbe assunto nei confronti della popolazione araba ${ }^{11}$.

La prima parte del volume si chiude con due capitoli dedicati, rispettivamente, alla Dichiarazione Balfour e al consolidamento della presenza ebraica in Palestina negli anni del mandato britannico (1920-1948), nei quali l'autore riconduce il sostegno della Gran Bretagna alla costituzione di uno stato ebraico a ragioni di politica estera ed economica ${ }^{12}$ e rilegge l'epoca della leadership di Ben Gurion e del predominio del sionismo socialista alla luce del "problema arabo", divenuto centrale a seguito dell'inasprirsi degli scontri tra coloni ebrei e popolazione araba e dell'emarginazione delle correnti del sionismo favorevoli alla creazione di uno stato bi-nazionale in Palestina.

La seconda parte del testo prende le mosse dall'approvazione della Risoluzione n. 181 da parte dell'Assemblea generale delle Nazioni unite (1947) per proporre una rilettura globale della storia dello Stato di Israele dalla prospettiva del confronto e scontro tra le diverse anime del sionismo.

Il primo nucleo di riflessioni concerne il «trattamento decisamente discriminatorio» ${ }^{13}$ riservato alla popolazione araba nei primi decenni di esistenza di Israele, che trovava fondamento nelle norme in vigore (la Dichiarazione di indipendenza e la nota "Legge del ritorno," che escludeva dall'attribuzione della cittadinanza israeliana coloro che non professassero la religione ebraica) e in provvedimenti che penalizzavano i cittadini arabi, imponendo loro un regime separato di tipo militare, abolito solo nel 1966.

Marzano dedica ampio spazio alla controversia storiografica sorta attorno alla "questione araba", citando, fra gli altri, Ilan Pappe, che ha accusato i governi israeliani di aver condotto una vera e propria "pulizia etnica" ${ }^{14}$, Benny Morris, secondo il quale l'esodo della popolazione araba dai territori israeliani fu dovuto a numerose concause, tra le quali la condotta delle élite palestinesi ${ }^{15}$, e Shira Robinson, che ha coniato l'efficace definizione di "stato liberal-coloniale" per descrivere il regime adottato in Israele ${ }^{6}$.

L'autore, evitando coerentemente di lasciarsi coinvolgere dalle polemiche scaturite dal confronto tra New Historians e storiografia tradizionale, si sofferma nuovamente sulle "possibilità mancate" da parte del sionismo, attraverso il riferimento alle voci di chi, come Hannah Arendt, si fece promotore della convivenza paritaria e pacifica tra popolazione ebraica ed araba ${ }^{14}$, destinate a rimanere sostanzialmente inascoltate da parte della classe al potere in Israele.

\footnotetext{
${ }^{11}$ PENSLAR, Derek J., «Zionism, colonialism and Postcolonialism», in Journal of Israeli History, 20, 2-3/2001, pp. 84-98.

${ }^{12}$ L'autore riprende un'interpretazione proposta in MARZANO, Arturo, «Il ruolo del petrolio nella politica britannica in Mesopotamia (1900-1920)», in Il Politico, 4/1996, pp. 629-650.

${ }^{13}$ MARZANO, Arturo, Storia dei sionismi, cit., p. 128.

${ }^{14}$ ARENDT, Hannah, Ebraismo e modernità, Milano, Unicopli, 1986 [Ed. originale: The Jew As Pariah. Jewish Identity and Politics in the Modern Age, New York, Grove Press, 1978].
} 
Il volume procede con un capitolo dedicato alla cesura storica determinata dalla vittoria di Israele nella Guerra dei sei giorni, che creò un clima propizio alla diffusione del sionismo religioso, chiuso alle rivendicazioni della popolazione araba, e alla colonizzazione della Cisgiordania, all'interno di «un progetto condiviso da ampi settori della politica, della cultura e della società israeliana», pur se accompagnato da una dura critica di stampo anti-sionista ${ }^{15}$.

Segue un esame di un altro momento chiave della storia di Israele, la vittoria alle elezioni legislative del 1977 da parte del Likud, partito della destra erede del sionismo revisionista, che diede nuovo impulso alla colonizzazione dei Territori palestinesi e al «progressivo sdoganamento di una retorica che non nascondeva più il proprio razzismo nei confronti degli arabi» ${ }^{16}$.

Il volume si conclude con l'esame delle pagine più recenti della storia di Israele: gli Accordi di Oslo (1993) e l'attività politica di Yitzhak Rabin, che ne fu il promotore, sono pertanto ricondotti ad una declinazione del sionismo che accordava la priorità alla sicurezza dello Stato di Israele, indipendentemente dalle eventuali variazione dei suoi confini, contrapposta ad una corrente che considerava irrinunciabile il mantenimento dei Territori occupati, che sarebbe prevalsa con il ritorno al potere del Likud.

Le vicende attraversate da Israele nell'ultimo ventennio, dall'omicidio di Rabin all'approvazione del protocollo di Hebron (1997), fino alla stipula degli accordi di Annapolis (2007), sono rievocate al fine di introdurre un ultimo capitolo, certamente il più "difficile" da un punto di vista storiografico, nel quale l'autore ritrae il sionismo dei nostri giorni. In queste pagine Marzano opera una distinzione tra corrente autoritaria, incarnata dal premier Benjamin Netanyahu, "sionismo critico", al quale afferiscono coloro che contestano in maniera più o meno radicale diversi aspetti della storia della nazione ebraica, e pensiero post-sionista, secondo il quale il modello di Stato ebraico sinora adottato da Israele dovrebbe evolversi in un ordinamento "alternativo", per citare il titolo di un noto articolo di Tony Judt, basato sulla piena parità di diritti tra popolazione araba ed ebraica ${ }^{17}$, evitando di cadere nella trappola di coloro che cercano di mettere a tacere l'opposizione all'attuale condotta politica dei governi israeliani accusandola di antisemitismo.

In conclusione del volume Marzano, pur soffermandosi sulla gravità della crisi politica del Medio Oriente e sulle contraddizioni nelle quali si dibatte il sionismo contemporaneo, propone argomenti a favore di una possibile, pur se complessa, normalizzazione dei rapporti tra popolazione ebraica ed araba, attribuendo al suo lavoro storiografico una funzione civile che arricchisce ulteriormente il valore dell'opera.

\footnotetext{
${ }^{15}$ MARZANO, Arturo, Storia dei sionismi, cit., p. 149.

${ }^{16}$ Ibidem, p. 164

${ }_{17}$ JUDT, Tony, «Israel: The Alternative», in The New York Review of Books, 23 ottobre 2003, URL: < http://www.nybooks.com/articles/2003/10/23/israel-the-alternative/ > [consultato il 7 dicembre 2017].
} 
Nell'insieme, dunque, il volume potrà interessare un pubblico che non sia limitato ai soli specialisti ed essere utilizzato a scopi didattici, sebbene non includa un apparato documentario che avrebbe potuto agevolare la lettura da parte di chi non ha una consuetudine di lunga data con i temi trattati (sono presenti, tuttavia, numerosi riferimenti a documenti e fonti disponibili online).

In conclusione, la Storia dei sionismi di Arturo Marzano, mettendo a frutto l'ampia conoscenza della letteratura esistente in una trattazione originale e, per certi versi, controcorrente, è destinata a divenire un punto di riferimento nell'attuale panorama di studi, fornendo materiali e suggestioni per future ricerche su questo movimento. 


\section{L'AUTORE}

Elisa TIZZONI (La Spezia, 1983), PhD in Storia contemporanea, ha svolto incarichi di ricerca e didattica presso le Università di Pisa, Firenze, Nizza, Salisburgo, e presso diverse istituzioni culturali. Nel 2017 ha ottenuto una borsa di ricerca Postgraduate Vibeke Sørensen Grant presso l'Historical Archives of the European Union a Firenze. Le sue pubblicazioni comprendono oltre una trentina di articoli e saggi in italiano, francese e inglese, principalmente nell'ambito della storia del turismo.

URL: < http://www.studistorici.com/progett/autori/\#Tizzoni > 